\title{
Semiclassical Formulation of Optimal Control Theory
}

\author{
KONDORSKIY Alexey ${ }^{1,2}$, MIL'NIKOV Gennady ${ }^{3}$ and NAKAMURA Hiroki ${ }^{4}$ \\ ${ }^{1}$ Institute for Molecular Science, Okazaki 444-8585, Japan \\ ${ }^{2}$ Lebedev Physical Institute, Leninsky pr., 53, Moscow, 119991, Russia \\ ${ }^{3}$ Institute for Structural Macrokinetics, Chernogolovka, Moscow Reg., 142432, Russia \\ ${ }^{4}$ The Graduate University for Advanced Studies, Okazaki 444-8585, Japan \\ (Received: 5 October 2004 / Accepted: 24 May 2005)
}

\begin{abstract}
In the present paper semiclassical formulation of optimal control theory is made by combining the conjugate gradient search method with new approximate semiclassical expressions for correlation function. Two expressions for correlation function are derived. The simpler one requires calculations of coordinates and momenta of classical trajectories only. The second one requires extra calculation of common semiclassical quantities; as a result additional quantum effects can be taken into account. The efficiency of the method is demonstrated by controlling nuclear wave packet motion in a two-dimensional model system.
\end{abstract}

\section{Keywords:}

optimal control theory, laser control, semiclassical approximation

Controlling molecular processes by laser pulses is a subject of active research in physics. One of the most natural and flexible approaches in this area is the optimal control theory (OCT) [1,2]. It is based on the idea that the controlling laser pulse should maximize a certain functional so that the variational principle can be used to design the pulse. The procedure leads to a set of equations for optimal laser field, which includes two Schrodinger equations to describe the dynamics starting from the initial and target state wave packets. The optimal laser field is given by the imaginary part of the correlation function of these two wave packets. This system of equations of optimal control must be solved iteratively in general.

During the last 20 years a number of methods based on this idea have been developed [3-9]. The earliest formulations of the problem for classical [3-6] and quantum [7] systems employ the well established numerical conjugate gradient search method to solve the system of equations iteratively and maximize the certain functional. Later the more effective numerical schemes to solve this system of optimal control equations have been introduced $[8,9]$. These iterative algorithms converge faster than the gradient-type.

In all these approaches the optimal laser field is given by the imaginary part of a certain correlation function. In the simple case of quantum conjugate gradient search method [7] this function has the form

$$
\boldsymbol{\Theta}(t)=\langle\phi(t)|\boldsymbol{\mu}(\boldsymbol{r})| \chi(t)\rangle,
$$

where $|\phi(t)\rangle$ and $\langle\chi(t)\rangle$ are the wave packets driven by the optimal field starting from different initial states and $\boldsymbol{\mu}(\boldsymbol{r})$ is the dipole moment. To calculate the optimal field two Schrodinger equations for $|\phi(t)\rangle$ and $\langle\chi(t)\rangle$ together with the equation for correlation function (1) should be solved iteratively in general; hence its numerical cost becomes huge for multi-dimensional systems.

Since the quantum OCT is limited to low-dimensional systems because of formidable numerical cost, it is strongly desired to incorporate semiclassical approaches of wave packet propagation like the HermanKluk method $[10,11]$ into the OCT. Within the semiclassical approach each wave packet is formed by summation of contributions from a large number of classical trajectories. In order to calculate the correlation function the double summation with respect to this large number of trajectories should be performed at each time step. These cause significant numerical difficulties in direct incorporation of semiclassical approaches into the OCT.

In the present work semiclassical formulation of optimal control theory is made by combining conjugate gradient search method with new approximate semiclassical expressions for correlation function [12]. Approximate analytical evaluation of one of the sums is performed by direct linearization of semiclassical Herman-Kluk propagator. Two approximate semiclassical expressions for correlation function, which con-

Corresponding author's e-mail: akondor@ims.ac.jp 


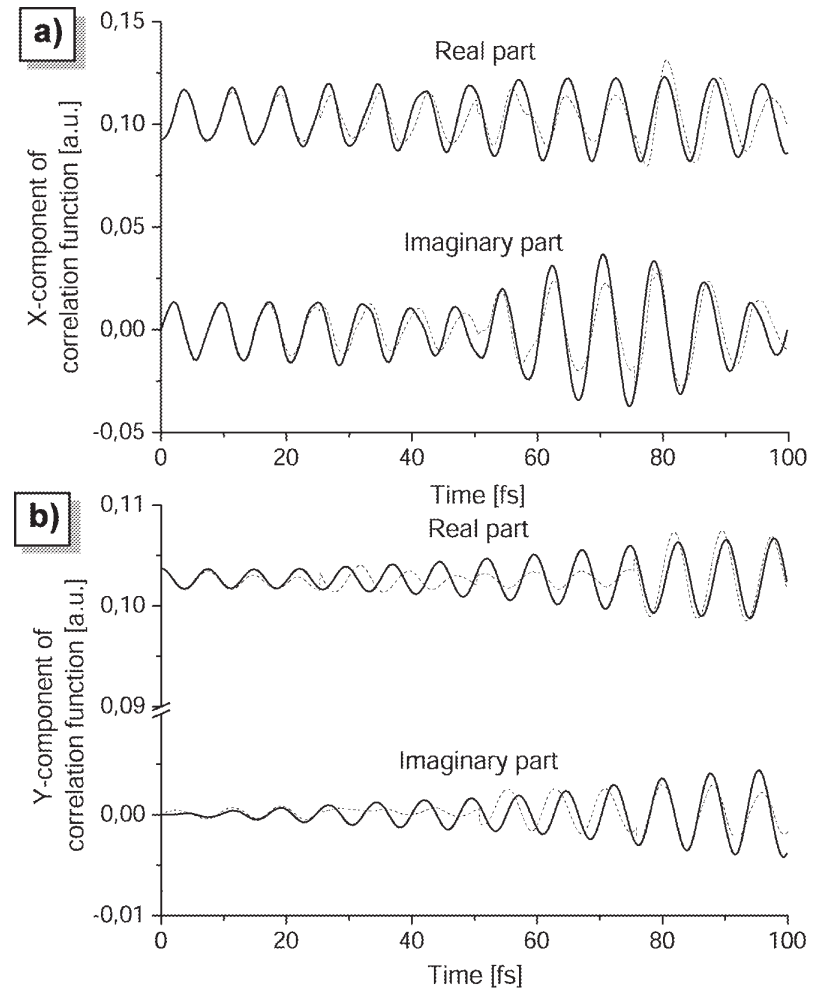

Fig. 1 X-component (a) and Y-component (b) of correlation function of initial state and target state wave packets (Eq. (1)). Bold line: exact quantum result. Dash line: semiclassical results. The initial state is a ground state wave packet. The target state is a same shape wave packet shifted for 0.7 a.u. in $\mathrm{O}-\mathrm{H}$ bond direction.

tain only one summation with respect to trajectories, are obtained. These expressions do not contain the Herman-Kluk prefactors and exponential factors, which also simplify the calculations very much.

Semiclassical results obtained for correlation function are found to be in good agreement with the exact quantum one for about $5-6$ oscillations of real and imaginary part of correlation function or for about 200 time integration steps (See Fig. 1). The accuracy of our semiclassical results is deteriorated naturally at long time durations. To improve the applicability of the approach it is good enough to recalculate the coefficients used in our formula from time to time.

The efficiency of the method is demonstrated by controlling nuclear wave packet motion in a twodimensional HOD model system. The following three important processes are controlled: (i) shift of the ground state wave packet, (ii) uniform acceleration of the ground state wave packet and (iii) $\mathrm{H}+\mathrm{OD} \rightarrow \mathrm{HO}+$ $\mathrm{D}$ reaction dynamics (See Fig. 2). High final absolute values of the overlap of target state wave packets and final wave packets are achieved: about $90-95 \%$ for first two processes and $75-80 \%$ for the last one.

The computational time of quantum wave packet

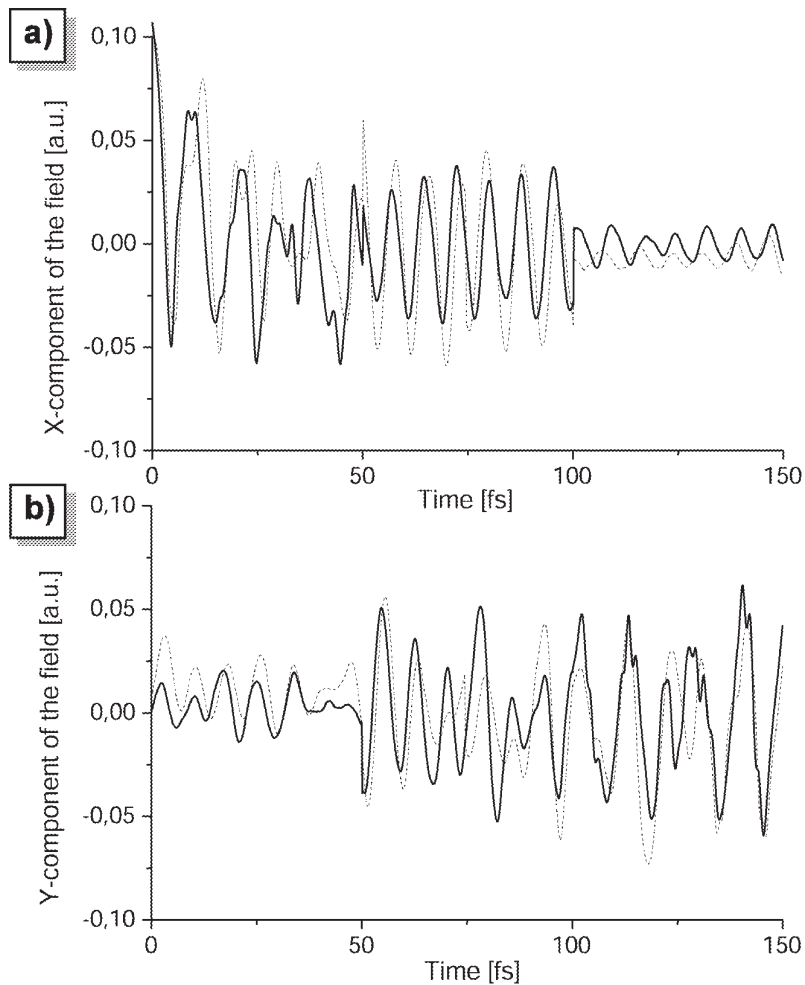

Fig. 2 X-component (a) and Y-component (b) of the optimal field obtained for model $\mathrm{H}+\mathrm{OD} \rightarrow \mathrm{HO}+\mathrm{D}$ reaction. Solid line: exact quantum result. Dash line: semiclassical results.

propagation grows exponentially with dimensionality, so it becomes huge for multi-dimensional systems. On the other hand, the semiclassical approaches make it possible to reproduce almost all quantum effects with its computational cost not growing rapidly as the dimensionality of the system increases (about as square of dimensionality). This means that the present semiclassical theory is expected to be much more efficient than the quantum in the case of higher dimensions.

\section{References}

[1] S.A. Rice and M. Zhao, Optical Control of Molecular Dynamics (John-Willey \& Sons 2000).

[2] S.M. Hurley and A.W. Castleman, Science 292, 648 (2001).

[3] A. Peirce, M. Dahleh and H. Rabitz, Phys. Rev. A 37, 4950 (1988).

[4] S. Shi, A. Woody and H. Rabitz, J. Chem. Phys. 88, 6870 (1988).

[5] C. Schwieters and H. Rabitz, Phys. Rev. A 44, 5224 (1991); ibid. 48, 2549 (1993).

[6] J. Botina, H. Rabitz and N. Rahman, J. Chem. Phys. 102, 226 (1995).

[7] R. Kosloff, S. Rice, P. Gaspard, S. Tersigni and D. Tannor, Chem. Phys. 139, 201 (1989).

[8] J. Somloi, V. Kazakov and D. Tannor, Chem. 
Phys. 172, 85 (1993).

[9] W. Zhu, J. Botina and H. Rabitz, J. Chem. Phys. 108, 1953 (1998).

[10] M. Herman and E. Kluk, Chem. Phys. 91, 27 (1984).
[11] E. Kluk, M. Herman and H. Davis, J. Chem. Phys. 84, 326 (1986).

[12] A. Kondorskiy and H. Nakamura, J. Theor. Comput. Chem. (Special Issue for the proceedings of the 1st APACTC) (2004) (in press). 